\title{
Interrogatoire du mort en pays joola felup
}

Questioning the deceased (joola-felup)

Odile Journet et André Julliard

\section{(2) OpenEdition}

Journals

Édition électronique

URL : http://journals.openedition.org/span/1141

DOI : 10.4000/span. 1141

ISSN : 2268-1558

\section{Éditeur}

École pratique des hautes études. Sciences humaines

\section{Édition imprimée}

Date de publication : 1 novembre 1989

Pagination : 135-153

ISSN : 0294-7080

\section{Référence électronique}

Odile Journet et André Julliard, «Interrogatoire du mort en pays joola felup », Systèmes de pensée en Afrique noire [En ligne], 9 | 1989, mis en ligne le 15 octobre 2013, consulté le 01 mai 2019. URL : http:// journals.openedition.org/span/1141; DOI : 10.4000/span.1141 


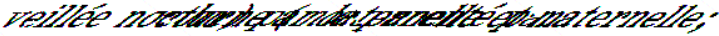

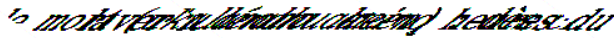

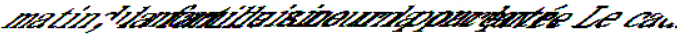

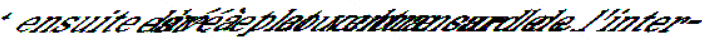

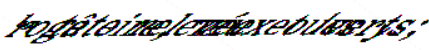

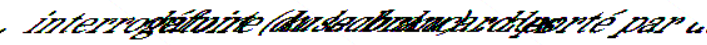

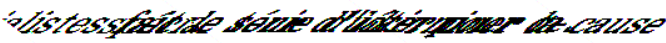
de los mart,

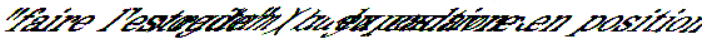

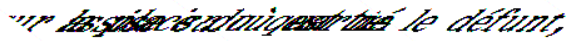

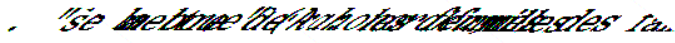

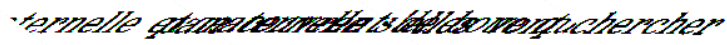

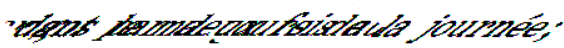

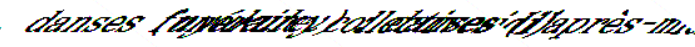

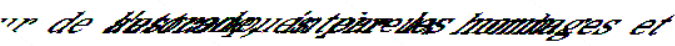

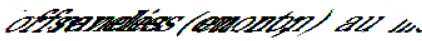

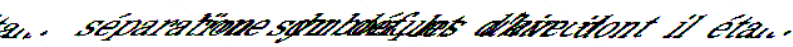
rablequacmonombel,

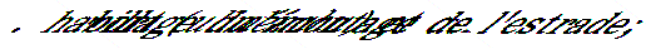

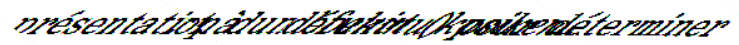

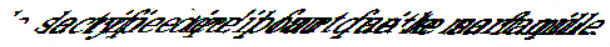
en lo tersmatisyons

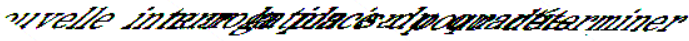

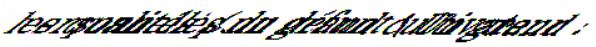
nitteur,' etcel,

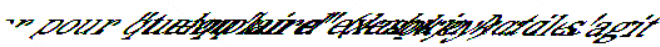

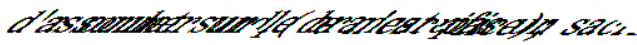

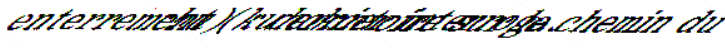

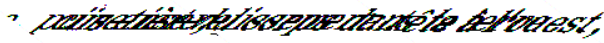

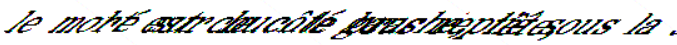

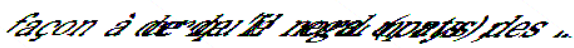

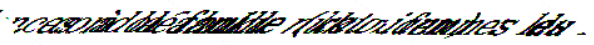

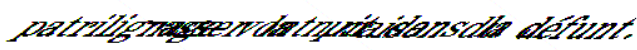

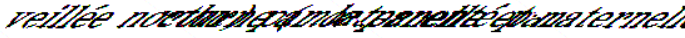

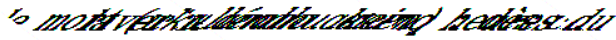

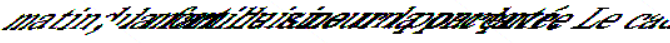

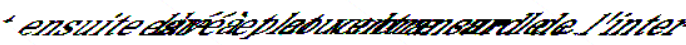

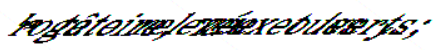

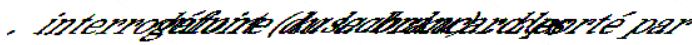

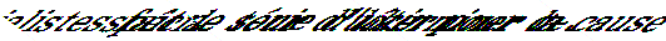
de la mart,

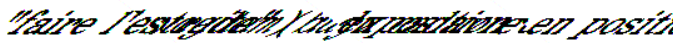

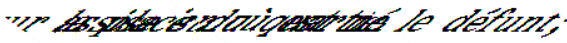

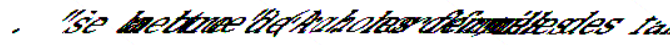

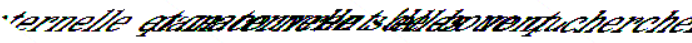

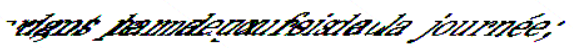

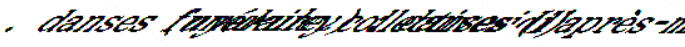

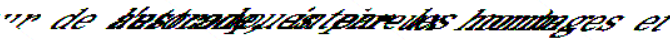

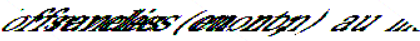

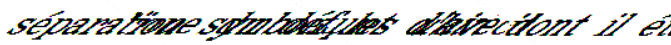

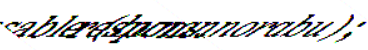

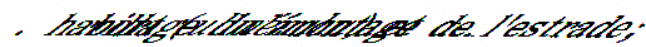

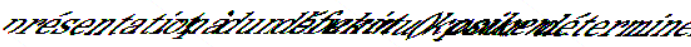

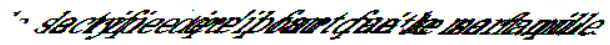
en la tairantistans

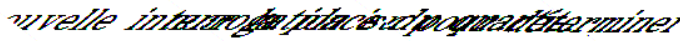

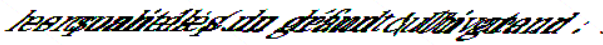
luttewn,' ets:-

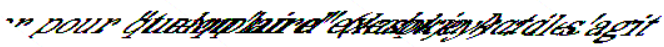

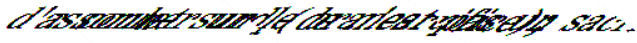

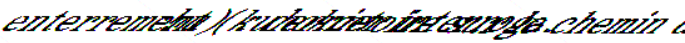

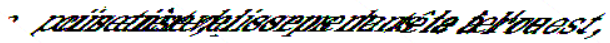

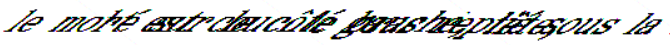

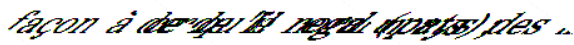

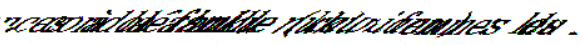

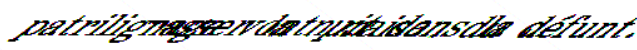


Apparemment, il n'y a pas d'interrogateurs désignés : tous ceux qui se sentent concernés ou pensent être impliqués dans l'événement de ce décès peuvent prendre la parole. L'interrogatoire prend la forme d'un dialogue dans lequel chacun exprime sa version de l'événement, ses explications, son opinion et ses questions. Par l'intermédiaire du brancard, le mort "répond" en ayant à sa disposition cinq types de comportements-réponses :

1. pour une réponse positive (notée : "oui" dans le compte rendu de l'interrogatoire), le défunt "poussera" le brancard vers son interlocuteur, qui l'arrêtera en saisissant de la main droite le barreau transversal sur lequel repose la tête du mort. Puis, il fera le geste de le repousser au centre, signifiant ainsi qu'il accepte la réponse du défunt. Si l'interrogateur refuse ce "oui" - signifiant ainsi que le mort ment - il s'écartera du brancard qui entre dans la foule, laquelle se disperse aussitôt. Le brancard retourne ensuite au centre de la place.

Le mort peut également souligner l'importance de sa réponse (noté : "oui"), en "fonçant" littéralement sur le questionneur, qui devra faire un effort physique réel - et même quelquefois appeler à l'aide son voisin - pour renvoyer le brancard. Ce comportement donne une orientation décisive aux questions ultérieures;

2. pour une réponse négative (notée : "non"), le brancard reste immobile au centre de la place;

3. le défunt peut également refuser de répondre (noté : ".."); les porteurs pratiquent alors un mouvement de balancement latéral de peu d'amplitude. (Piétiner sur place en se balançant est une attitude courante pour exprimer "la honte" d'avoir commis une faute envers le code moral en usage dans la société felup);

4. enfin, le mort peut choisir son interlocuteur, en se dirigeant droit sur lui. Cette situation se produit dans un moment où chacun reste silencieux et où l'interrogatoire ne progresse plus. 
Sikundo (féticheur, cousin paternel du défunt Osile; il était appelé oncle par ce dernier parce que plus âgé que lui):

- "Je ne peux pas tout dire ce qui t'a tué. Tu nous a montré les gens qui savent pourquoi tu es mort. Peut-être ton âme est-elle dans le boekin?"

- "Non"

Le féticheur demande à Osile si, de son vivant, il a accompli toutes les obligations rituelles dues à Ekobey. Il cherche à savoir si la mort ne serait pas un châtiment du boekin: le boekin aurait littéralement dévoré le mort.

- "Est-ce que ton âme n'est pas dans Ekobey?"

- "..."

- "Il faut que tu nous dises si réellement tu as eu des discussions avec Ekobey?"

- "Oui"

Avoir des discussions avec le boekin est une formule ambiguë, même pour les deux personnes qui nous ont aidé à traduire l'interrogatoire. Elle peut signifier d'abord qu'Osile était en conflit avec le boekin, soit parce qu'il avait refusé d'accomplir un sacrifice (une rébellion en quelque sorte), soit parce qu'il avait négligé ou oublié de le faire: le sacrifice représente toujours une charge économique et un endettement prévisible. La question peut aussi implicitement signifier : Osile n'est-il pas un de ces "hommes mauvais" qui utilise le boekin pour ensorceler ses victimes. A cette ambiguïté répond une hésitation du brancard: elle signifie la honte sociale (surtout pour la famille) d'avouer cette relation.

Le brancard recule et se dirige vers Sikahunan (cousin paternel de Osile, habitant la même concession).

\section{Sikundo :}

- "C'est chez Sikahunan que tu as pris la mort. Sikahunan perdait ses enfants. C'est toi qui les a tués?"

- "Oui"

Dans les années 68-70 - presque 20 ans en arrière - Sikahunan a été la victime de Sihongoli, le père d'Osile, qui était un puissant féticheur de Susanna et l'un des gardiens du boekin Ekobey. Pourquoi devient-il le sorcier de son neveu? Parce que celui-ci, s'étant converti au catholicisme, refusait de subir le rite de la circoncision. Sikahunan échappera à son oncle après un mois de maladie mais, et jusqu'à sa mort, Sihongoli le poursuivra, notamment après son mariage catholique, en tuant (en "prenant") les deux premiers enfants du couple. 
Le brancard fait le tour de la place et s'arrête en direction du boekin Ekobey

- "C'est Ekobey qui t'a tué ?"

- "Oui"

- "Est-ce que tu as eu des disputes avec ton frère ?"

- "Non"

(Sikahunan et Osile sont cousins de même génération : ils sont appelés frères).

- "Sikahunan a perdu ses enfants, c'est toi qui les a mangés ?"

- "Oui"

- "Tu as raison! Tu as bien fait de manger les enfants de ton frère plutôt que de manger les enfants d'autrui. C'est pour cela que tu as eu des problèmes avec Ekobey !"

- "Oui"

- "Tu as bien fait de prendre les enfants de la maison plutôt que d'aller ailleurs. Sinon, je t'aurais demandé : "pourquoi es-tu allé dans une autre maison manger les enfants d'autrui ?"

Malum exprime une opinion très appréciée de l'assistance car elle va dans le sens de l'apaisement et non du conflit toujours redouté : d'une part, il rappelle habilement qu'un sorcier n'est pas entièrement responsable de ses actes (il est poussé) et d'autre part, il démontre que son cousin est resté dans une certaine norme sociale en n'attaquant pas des étrangers à son lignage, évitant ainsi de déclencher des conflits pouvant dégénérer en violence (poison, bataille, etc.).

Usumboal (féticheur, gardien de Ekobey) :

- "C'est donc toi qui t'es tué, ce n'est pas quelqu'un qui t'a mangé. C'est toi-même qui t'es fait tuer. Le kasaabaku ne sera pas long!"

Le brancard ne répond ni oui ni non, et il ne refuse pas non plus la question : il l'ignore en retournant lentement au centre de la place. Le comportement du brancard se comprend car l'intervention de Usumboal sert avant tout à marquer son extériorité par rapport à la relation existant entre son boekin et Osile : ce que personne ne mettrait en doute compte tenu de la notoriété de Usumboal.

Abayan (cousin paternel) :

- "Pourquoi as-tu répondu à Sikundo et n'as-tu pas voulu répondre à Malum ? Tu as bien répondu à Usumboal ? Ton père, lui, ne va pas te demander de quoi tu es mort !" 


\section{Usumboal}

- "Nos frères t'ont interrogé pour savoir si tu es mort d'avoir tué les enfants de notre frère. Est-ce bien vrai ?"

- "Oui"

- "C'est vrai, notre frère a perdu ses enfants. Personne ne dira le contraire. Nous parlons de la même chose depuis le début".

- "Non"

- "Tu ne veux pas répondre parce que j'ai dit à tout le monde de quoi tu es mort?

- "Non"

- "Nous, nous avons l'habitude de nous appuyer sur ce que chacun de nous a dit. Nous allons donc reprendre ces paroles pour t'interroger !"

Toutes les questions à venir devront se centrer sur les deux énoncés suivants:

1) Osile est mort parce qu'il a tué les enfants de ses cousins;

2) Il a été tué par le boekin Ekobey.

- "C'est toi qui a tué les enfants de tes frères, et c'est pour cela que tu es mort?"

- "Oui"

- "Sikundo t'a demandé où vas-tu manger?

C'est ici (= dans la concession d'Osile) qu'il faut manger !"

- "Oui"

- "Comme Ekobey a refusé (que tu manges les enfants), pourquoi es-tu allé manger? Est-ce que les hommes ne passent pas à l'interrogatoire ?"

- "Bon, tu dis que l'interrogatoire (celui de Usumboal) est fini ! L'interrogatoire est fini?"

- "Oui"

Ampahanji, le beau-père d'un cousin d'Osile chez qui ce dernier allait souvent jouer lorsqu'il était enfant, veut parler mais le brancard part dans la direction opposée. 
- "Tes frères ne t'ont pas mangé et toi tu es mort d'avoir mangé leurs enfants ?"

"Oui +"

- "Le sorcier meurt, l'âme aussi meurt. Quand Osile était malade, nous étions partis en réunion à Kugel, je vous ai dit : "je ne garde pas le pagne pour moi, mais je garde ça pour Osile parce qu'Osile c'est moi qui l'ai mis au monde". Parce que moi toute l'année je suis malade, donc je ne crois pas que je vais mourir maintenant".

Ampahanji s'était vraisemblablement rendu à Kugel, quartier de Susana, à l'une de ces nombreuses réunions où se discutent les problèmes du village, et $y$ avait montré le pagne qu'il venait d'acheter en prévision du décès d'Osile (offrande).

\section{Le brancard reste devant lui}

- "Moi et Sihonguli : qui devait mourir le premier ? Or, Sihonguli est mort avant moi."

Ampahanji veut dire qu'avec Sihonguli, ils sont comme les deux parties complémentaires d'un tout : si l'un est malade, l'autre tombera malade pour que le premier guérisse (explication donnée par les deux traducteurs de l'interrogatoire). Une telle complémentarité s'observe souvent chez les Felup, entre deux grands lutteurs.

Cette remarque donne tout son sens à l'affirmation de Ampahanji: "je suis malade toute l'année, mais je sais que je ne mourrais pas", puisqu'Osile, représentant de son père (représentation encore renforcée par la présence du caractère héréditaire de sorcier) est mort une fois encore avant lui.

- "Kia Osile !" (Expression de pitié).

\section{La mère d'Osile}

- "Awania ("awania" = grand cultivateur), tu as vu ton interrogatoire!"

- "Oui +"

- "Quand je lui demandai de son vivant l'origine de sa maladie, je savais que le jour où il mourrait, il me dirait qui l'a tué. Voilà aujourd'hui que tu te diriges vers ton frère : tu t'es disputé avec lui ?

Vous vous êtes disputés dans les rizières. Quand Sikahunan a cultivé sa parcelle, Osile a dit que c'était la sienne, et il a arraché tout le riz que Sikahunan avait repiqué, et il a planté son propre 


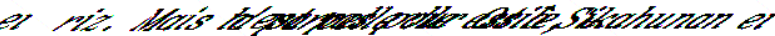

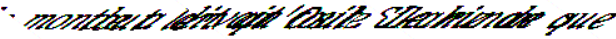

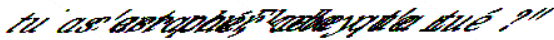
"van" - "

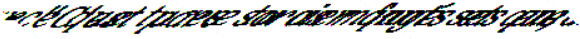
the mort?

"

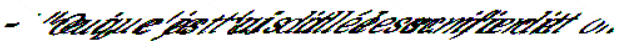

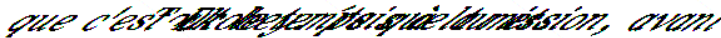

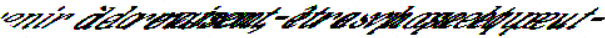

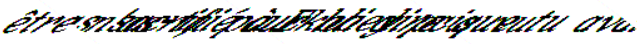

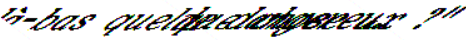

- "Non"

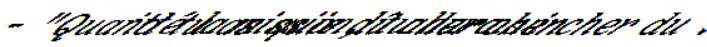

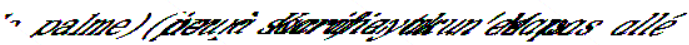

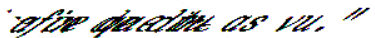

- "Nan"

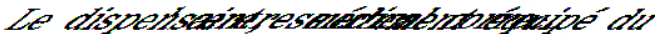

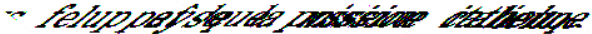

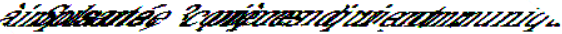

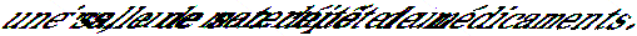

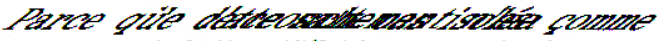

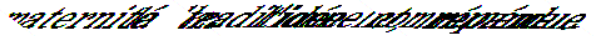

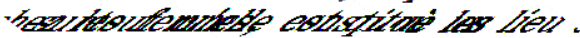

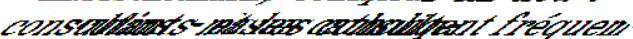

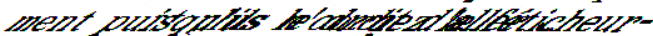

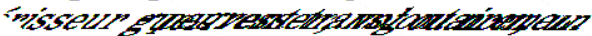

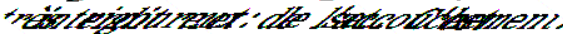

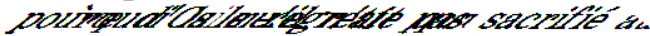

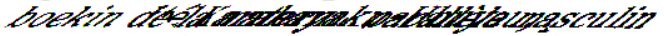

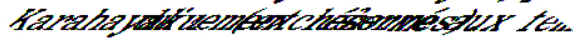

\section{Toune diasile}

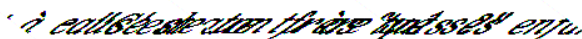

- "OMI't"

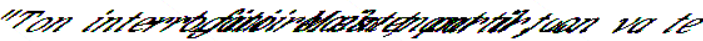

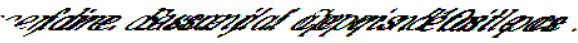

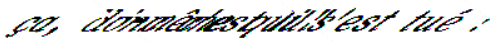

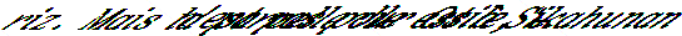

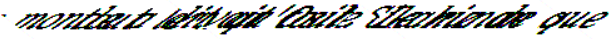

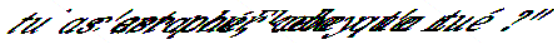

"Von" - "

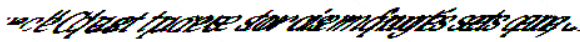

thes mont?

"

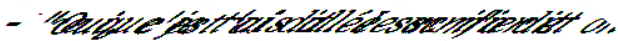

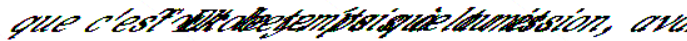

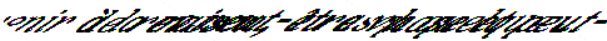

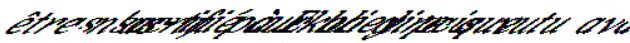
"bas quelumentarewn, p"

- "von"

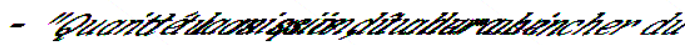

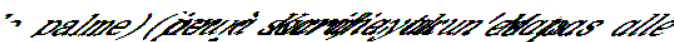

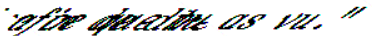

- "Non"

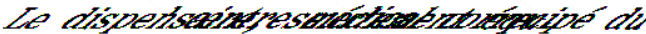

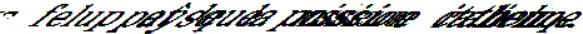

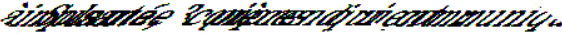

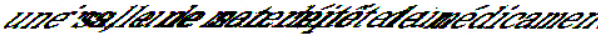

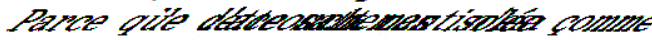

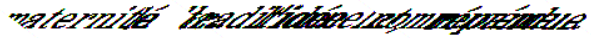

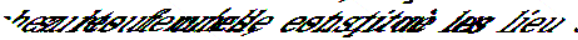

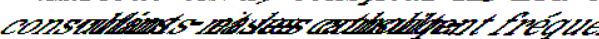

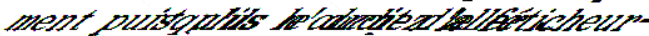

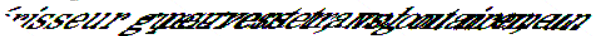

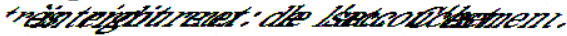

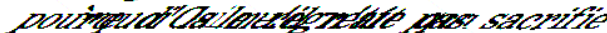

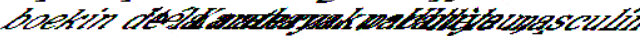

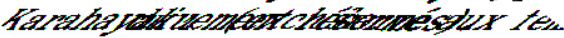

To mine dinste

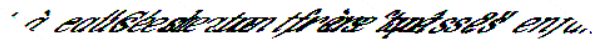

- "OMI'T"

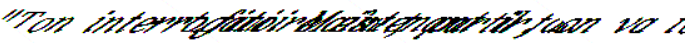

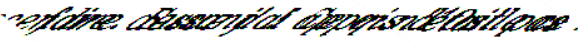

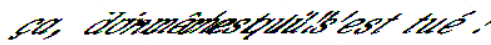


resté, et c'est là que je l'ai aperçu qui partait dans les rizières. Sikahunan était donc fâché contre toi parce que tu lui avais fait quelque chose ?"

Sikahunan se cachait car en tant que catholique vivant à Santa Maria, il ne voulait pas se rendre à la convocation du féticheur, craignant réellement pour sa vie : Osile, même malade, restait menaçant d'autant qu'en cette occasion, Sibekandopa pouvait être son allié.

- "Oui"

- "Ce que tu lui a fait ne lui plaît pas, c'est pour cela qu'il n'est pas venu te répondre. C'est bien à cause de ton frère que tu es mort ?"

- "Oui"

Remarquons que Sibekandopa préfère interpréter l'attitude de Sikahunan comme une démonstration d'irritation et non de méfiance envers eux : paroles qui vont dans le sens de l'apaisement de ces conflits, objectif général de l'interrogatoire.

- "C'est la seule chose que tu as faite, ou bien il y en a encore une autre ?"

- "Oui +"

Sijula (beau-frère d'Osile) :

- "Si mon grand frère était là, moi je ne devrais pas parler ! C'est mon grand frère Kantente qui devrait parler. C'est donc comme tes frères te l'ont demandé que tu es mort? A cause de ton frère ?"

- "Oui"

- "Ce n'est pas à cause de ta mère ?"

- "Non"

- "C'est toi-même qui t'es tué".

Le brancard se retourne pour partir.

- "Viens ici ! Est-ce que tu n'as pas entendu ce que ta mamam a dit ? Tu n'est pas mort à cause de la parcelle pour laquelle tu t'es disputé avec Sikahunan !"

- "Non"

- "Donc, c'est toi qui t'es tué !"

Le brancard se dirige vers la femme d'Osile. 
des enfants au monde puis tu meurs, tu les laisses dans les mains des autres".

\section{En entendant ces paroles, Sikundo va frapper la tête des deux enfants qui assistent aux funérailles.}

\section{La femme d'Osile}

- "Quand je me suis mariée avec Osile, on était bien, on ne s'est jamais disputé. Trois ans après le mariage, j'ai eu des problèmes avec lui et j'ai quitté la maison. Je suis allé à Ziguinchor (Sénégal). Osile m'a trouvé à Ziguinchor, il m'a dit qu'il a appris par les gens que des hommes m'ont cherchée en mariage. Mais Osile m'a dit : "Ne vas pas te marier ailleurs, retourne dans ma maison". Je suis revenue. Il a épousé une autre femme qui s'appelle Koutok. Koutok a eu un enfant la première, mais l'enfant n'était pas sur le dos de sa mère, il était sur mon dos. Quand elle a eu l'enfant, je l'avais appelée : "Koutok ?". Elle a répondu : "oui". "Celui-ci tu l'as mis au monde, mais ce n'est pas ton enfant, c'est mon enfant ! Quand tu auras un second, c'est celui-là qui sera ton enfant !"

Le brancard est relevé et revient au centre.

- "Mais voici mon enfant, toi qui t'es tué, tu as mangé les gens ?"

- "Oui +"

- "Comme ton papa est sorcier, toi aussi tu es sorcier?

Le brancard reste devant elle, puis recule au centre : Osile a "honte".

La première épouse d'Osile lance alors ce qui sera la dernière question :

- "Comme j'ai eu des enfants avec toi, es-tu content ?"

Le brancard court sur elle ("oui") et sans s'arrêter, traverse la foule pour reprendre le chemin qui mène au quartier de Kugel. 
Par conséquent, chaque interrogatoire engage quatre types de personnages : le défunt, les acteurs du drame mortuaire, le boekin et la communauté villageoise. La seconde phase du kasaabaku (qui sera sous le signe d'un autre boekin) réunira d'autres acteurs: tous ceux qui auront consulté la puissance en question et qui de ce fait sont considérés comme impliqués dans la mort d'Osile. Les questions et réponses sur les causes du décès ne feront que se répéter.

L'interrogatoire consiste certes à expliciter officiellement à proclamer publiquement en quelque sorte - les causes du décès que chacun connaît par avance. Mais il sert surtout à désamorcer les risques d'un éventuel cycle de violences (et pas seulement à travers la sorcellerie) qui pourrait s'enclencher après la mort d'Osile. Au cours des trois ou quatre interrogatoires dont se compose le kasaabaku, il sera répété que les frères et alliés de Sikahunan ne se vengeront pas sur les enfants ou la famille du mort. L'interrogatoire procure encore une autre sorte d'apaisement : il ramène subtilement, par le raisonnement moral, la cause du décès - aussi horrible soitelle - à la volonté de Dieu (ce qui n'exclut pas les obligations de sacrifices réparateurs pour la famille). 